\title{
INSIGHTS ON PP AND PS REFLECTION COEFFICIENTS AT POROELASTIC SEISMIC HORIZONS
}

\author{
José Sampaio de Oliveira and Jorge Leonardo Martins
}

\begin{abstract}
In oil and gas exploration, the seismic reflection method aims at obtaining information from subsurface and generating geological models based on travel times and amplitudes of recorded seismograms. In this context the poroelasticity theory is generally used as a good approximation for studying the seismic response of oil and gas reservoirs. As the reflection coefficients in poroelastic media have a very complex mathematical formulation, the dependence of such coefficients on fundamental poroelastic and petrophysical parameters remains poorly investigated. In this paper, we study the dependence of poroelastic reflection coefficients on petrophysical (i.e., the physical properties of the media) and data acquisition (i.e., the frequency of investigation and the angle of incidence) parameters. In order to investigate such a dependence, we use geologic models having a descontinuity interface between a clastic reservoir overlying a calcareous rock. We then construct 3D plots displaying the magnitudes of fast P-wave $\left(R_{P P}\right)$ and converted S-wave $\left(R_{P S}\right)$ reflection coefficients in saturated poroelastic media as a function of two specific variables. As the main petrophysical property, we considered the reservoir porosity $\phi$ varying from 5 to $30 \%$. By assuming the angle of incidence $\theta$ as one of the variables of the dependence (i.e., $0^{\circ} \leq \theta \leq 40^{\circ}$ ), we indirectly investigate the influence of the source-to-receiver distance on the reflection coefficients. As for the frequency of investigation presumably carried by the seismic source, we selected the following interval: from 10 to $200 \mathrm{~Hz}$. Absolute differences between the correspondent poroelastic and purely elastic reflection coefficients provided a way to select the most important poroelastic parameters which similarly affect the elastic coefficients. The reservoir porosity showed to be the most important dependence parameter in the variation of the reflection coefficients, either in poroelastic or in purely elastic media.
\end{abstract}

Keywords: reflection coefficients, poroelasticity, porosity, frequency investigation, incidence angle.

RESUMO. Na área de exploração de petróleo e gás, o método de reflexão sísmica visa a obtenção de informações de subsuperfície e a construção de modelos geológicos com base no tempo de percurso e nas amplitudes dos sismogramas. Neste contexto, a teoria da poroelasticidade é geralmente usada como uma boa aproximação para o estudo da resposta sísmica de reservatórios de petróleo e gás. Como os coeficientes de reflexão em meios poroelásticos possuem uma formulação matemática muito complexa, a dependência de tais coeficientes sobre parâmetros poroelásticos e petrofísicos fundamentais permanece pouco estudada. Neste trabalho, estudamos a dependência dos coeficientes de reflexão poroelásticos sobre parâmetros petrofísicos (i.e., propriedades físicas dos meios) e de aquisição de dados (i.e., a frequência de investigação e 0 ângulo de incidência). A fim de investigar a tal dependência, usamos modelos geológicos com uma interface de descontinuidade entre um reservatório clástico sobreposto a uma rocha calcária. Então, construímos gráficos 3D representando as magnitudes dos coeficientes de reflexão para a onda $\mathrm{P}$ rápida $\left(R_{P P}\right)$ e para a onda $\mathrm{S}$ convertida $\left(R_{P S}\right)$ em meios poroelásticos saturados em função de duas variáveis especíiticas. Consideramos a porosidade $\phi$ do reservatório como a principal propriedade petrofísica, variando de 5 a 30\%. Ao assumir 0 ângulo de incidência $\theta$ como uma das variáveis da dependência (i.e., $0^{\circ} \leq \theta \leq 40^{\circ}$ ), investigamos indiretamente a influência da distância da fonte ao receptor sobre os coeficientes de refleão. Quanto à frequência de investigação presumivelmente transportada pela fonte sísmica, selecionamos o seguinte intervalo: de 10 a $200 \mathrm{~Hz}$. As diferenças absolutas entre os correspondentes coeficientes de reflexão poroelásticos e puramente elásticos fornecem uma maneira de selecionar os parâmetros poroelásticos mais importantes que afetam de forma semelhante os coeficientes elásticos. A porosidade do reservatório mostrou ser o parâmetro mais importante na dependência dos coeficientes de reflexão, seja em meios poroelásticos ou em meios puramente elásticos.

Palavras-chave: coeficientes de reflexão, poroelasticidade, porosidade, frequência de investigação, ângulo de incidência.

Coordenação da Área de Geofísica, Observatório Nacional, Ministério da Ciência, Tecnologia \& Inovação, Rua General José Cristino, 77, São Cristóvão, 20921-400 Rio de Janeiro, RJ, Brazil. Phone: +55(21) 3504-9233; Fax: +55(21) 2580-7081 - E-mails: sampaio@on.br; jlmartins@on.br 


\section{INTRODUCTION}

Amplitude-versus-offset (AV0) analysis represents a very important technique applied to oil and gas exploration. To implement an AVO analysis, use of a suitable approximation for the reflection coefficient is fundamental (Shuey, 1985; Wang, 1999). In this way, several parameterization for the reflection coefficient can be obtained. Bortfeld (1961) applied an approximation that combined distinct elastic properties and are prone to AVO inversion. Ostrander (1984) showed that gas-related amplitudes anomalies can be studied using a reflection coefficient approximation as a function of Poisson's ratio. In order to incorporate effects of seismic anisotropy, Vavryčuk \& Pšenčýk (1998) derived approximate expressions for reflection coefficients at plane interfaces separating two media with generic anisotropy symmetry. Their approximation are expressed as a function of the Thomsen's anisotropy parameters $\epsilon, \gamma$ and $\delta$ which has intuitive sense, and are estimated in function of seismic velocities (Thomsen, 1986). In summary, different types of approaches can be used for describing reflection coefficients at an interface separating two geological media. Moreover, the initial assumptions for the media determine the structural complexity of the exact formula for the reflection coefficient. Classically, Zoeppritz (1919) considered both geological media as homogeneous elastic solids. However, homogeneous sedimentary layers, generally beddings, are better approximated as poroelastic materials (Biot, 1956), in view the premise of uniform distribution of fluid-saturated porous rocks. For this case the poroelastic constitutive model provides a better description of stresses-strain fields based on inclusion of parameters of the medium. Since then, other investigations have been developed in order to improve the knowledge of the influence of elastic and poroelastic theories on seismic reflection amplitudes (Bortfeld, 1961; Biot, 1962).

A good tutorial for the formulation for the exact isotropic elastic reflection coefficients can be found, for instance, in Aki \& Richards (1980) and Ursin \& Tjäland (1996). Basically, the steps for investigating exact reflection coefficients are:

(1) assumption for the properties of the media,

(2) description of all waves in terms of plane waves,

(3) definition of the boundary conditions, and

(4) solving the resultant algebraic linear system of equations arising from the analysis.

The same steps can be followed for any type of assumption on the properties of the media involved in reflection coefficient problem. Nevertheless, the more complicated are both media (i.e., isotropic elastic, anisotropic elastic or isotropic poroelastic), the more specific are the boundary conditions. For an incident wave at an interface separating two isotropic poroelastic media, Deresiewicz \& Skalak (1963) proposed a set of six equations as boundary conditions. In order to refine this set of equations, Sharma (2008) derived a correction which represents the fraction of interconnected porous at the discontinuous interface separating the porous layers. Such an imperfection in the interface can represent slipping, consequently, degradation of part of the strain energy. Using the boundary conditions proposed by Deresiewicz \& Skalak (1963) in addition with systems of open and sealed pores, Dennemann et al. (2002) obtained closed-form expressions for the reflection and transmission coefficients considering an incident wave at an interface separating an inviscid fluid and an isotropic poroelastic medium. Their work showed the complexity in the treatment of the reflection coefficient problem for poroelastic media in the case of a frequency-dependent investigation. Similarly, considering two isotropic poroelastic layers separated by a discontinuity interface, Gurevich et al. (2004) defined reflection and transmission coefficients for a normal incidence P-wave. In this instance, the reflection coefficients for fast and slow compressional waves are presented in simple forms supporting the limitation of near offsets. Using the seismic speeds and attenuation models proposed by White (1975), Quintal et al. (2011) qualitatively studied how fluid saturation can affect reflection coefficients in poroelastic media.

In this work, we study the problem reflection coefficients due to an incident P-wave at an interface separating two isotropic poroelastic rocks. More precisely, we numerically investigate the dependence of the exact, fast P-wave $\left(R_{P P}\right)$ and converted Swave $\left(R_{P S}\right)$ reflection coefficients on some critical petrophysical and data acquisition parameters. For this research were investigated as petrophysical parameters lithology, density, permeability and porosity. However we suppose as petrophysical variable only the porosity $\phi$ from the upper medium, while the variable parameters of data acquisition are the dominant frequency of seismic investigation $f$ and the incidence angle $\theta$. In order to analyze the parameter dependence, we use geologic models having a descontinuity interface between a clastic reservoir overlying a calcareous rock. We then construct 3D plots displaying the magnitudes of the $R_{P P}$ and the $R_{P S}$ reflection coefficients in saturated poroelastic media as a function of two specific variables. Absolute differences between the correspondent poroelastic and purely elastic reflection coefficients provided a way to select the most important poroelastic parameters which similarly affect the elastic coefficient. The reservoir porosity showed to be the most critical parameter to estimate variation of the coefficients. 


\section{METHODOLOGY}

This section shows the basic steps for the mathematical formulation of exact reflection coefficients. The geological model is formed by a discontinuity interface separating two homogeneous isotropic, elastic and poroelastic, layers. Although the exact formula for the isotropic elastic reflection coefficient is structurally very complicated because of the great number of terms, useful approximations can be found in the literature. In this way the approximate formulae provide insights on the dependence of the reflection coefficients on petrophysical parameters. See, for instance, the classical linearized approach derived in Aki \& Richards (1980). On the other hand, assumption of poroelastic layer for the geological model under analysis makes the complexity of the exact formulae for reflection coefficients even greater. Due to such a complexity, below we indicate the steps for evaluating the coefficients, while calculations are accomplished numerically in the results section.

\section{Reflection coefficients for elastic media}

Using a set of four elastic equations, Zoeppritz (1919) described the energy partition of waves generated after the incident of a Pwave at a discontinuity interface separating two homogeneous isotropic elastic layers. The reflection coefficients depend on the properties of both media separated by the interface, namely, compressional- and shear-wave propagation velocities and densities. Here, the upper and lower layer are distinguished with index 1 and 2, respectively. Assuming an incident P-wave and for a wide range of incidences, Zoeppritz's equations can be obtained by applying the continuity conditions through the interface, as follows:

$$
\begin{aligned}
& \text { 1. } u_{1}^{z}=u_{2}^{z}, \\
& \text { 2. } u_{1}^{x}=u_{2}^{x} \text {, } \\
& \text { 3. } \tau_{1}^{z z}=\tau_{2}^{z z} \text {, and } \\
& \text { 4. } \tau_{1}^{z x}=\tau_{2}^{z x} \text {, }
\end{aligned}
$$

where the axes $z$ and $x$ are taken normal and parallel to interface, respectively. The conditions 1 and 2 describes normal and parallel particle displacements of the medium related to interface. The conditions 3 and 4 are used for representing the normal and tangential stresses at the interface. The conditions above must be considered as the contribution of a set of waves propagating through individual layers. That is, incident and reflected waves propagate in the upper layer, while transmitted waves propagate in the lower layer. In order to simplify the problem, the incidence is considered in the same plane of P-wave polarization. Hence, shear waves with horizontal polarization are neglected from the analysis. Formally, the particle displacement for the upper $u_{1}$ and lower $u_{2}$ layers are written in the form of harmonic waves:

$$
\begin{gathered}
u_{1}^{l}=A^{l} \exp [i w(\mathbf{p} \cdot \mathbf{x}-t)] \\
+\sum_{j=1}^{2} A^{l} R_{j} \exp [i w(\mathbf{p} \cdot \mathbf{x}-t)],
\end{gathered}
$$

and

$$
u_{2}^{l}=\sum_{j=1}^{2} A^{l} T_{j} \exp [i w(\mathbf{p} \cdot \mathbf{x}-t)],
$$

where $i$ stands for imaginary unit and $l=x, z$. In the upper layer, the full wave motion evaluated in Eq. (1) along directions $x$ and $z$ are combined by one incident $\mathrm{P}$-wave and two reflected waves (i.e, $j=1,2$ ) corresponding to one P-wave and one Swave. As Eq. (2) shows, only two transmitted waves (one compressional and one shear) contribute to the particle displacement in medium 2. The terms $A_{l}$ in Eqs. (1) and (2) are the amplitudes of correspondent waves. Vector $\mathbf{p}$ represents the normalized slowness, i.e, $\mathbf{N} / V$, where $\mathbf{N}, V$ and $\mathbf{x}$ are the direction of phase propagation, the phase velocity and the position vector with components $(x, z)$, respectively. The energy partition for the generated waves at the interface is represented by the reflection and transmission coefficients, $R_{j}$ and $T_{j}$, respectively. The conditions 3 and 4 must be expanded using the well-known Hooke's law:

$$
\tau_{k l}=\lambda \delta_{k l} e_{j j}+2 \mu e_{k l} .
$$

For a homogeneous isotropic elastic media, the stresses depends only on Lamé's parameters $\lambda$ and $\mu$. The terms $e_{j j}$ and $e_{k l}$ correspond to the dilatation and to the shear strain tensor. By inserting Eqs. (1-3) into the set of boundary conditions 1-4, the resulting algebraic system of linear equations can be written in matrix form as:

$$
\mathbf{A}_{m n} \mathbf{X}_{n}=\mathbf{Y}_{m}
$$

in which the factors of displacement-stress are represented by matrix $\mathbf{A}_{m n}$. The column vector $\mathbf{Y}_{m}$ contains the independent terms of the linear system that are associated with the incident wave. The excitation factors $R_{j}$ and $T_{j}$ form the column vector $\mathbf{X}_{n}$, representing the unknowns to be evaluated. In this paper, we analyze only two coefficients, namely, the PP-wave reflection coefficient $\left(R_{P P}\right)$ and the converted P-to-S reflection coefficient $\left(R_{P S}\right)$. In compact form, the exact expressions for calculating 
$R_{P P}$ and $R_{P S}$ are (Aki \& Richards, 1980; Ursin \& Tjäland, 1996):

$$
R_{P P}=\frac{A+B-C-D+E-F}{A+B+C+D+E+F}
$$

and

$$
R_{P S}=\frac{-(M+N)}{A+B+C+D+E+F} .
$$

The parameters of Eqs. (5) and (6) are defined as follows:

$$
\begin{aligned}
A & =q^{2} p^{2} P_{1} Q_{1} P_{2} Q_{2}, \\
B & =\rho_{1} \rho_{2} V_{S 1} V_{P 2} P_{1} Q_{2}, \\
C & =\rho_{1} \rho_{2} V_{P 1} V_{S 2} Q_{1} P_{2}, \\
D & =V_{P 1} V_{S 1} P_{2} Q_{2} Y^{2}, \\
E & =V_{P 2} V_{S 2} P_{1} Q_{1} X^{2} \\
F & =V_{P 1} V_{P 2} V_{S 1} V_{S 2} p^{2} Z^{2}, \\
M & =2 V_{P 1} p q P_{1} P_{2} Q_{2} Y \text { and } \\
N & =2 V_{P 1} p V_{P 2} V_{S 2} P_{1} X Z,
\end{aligned}
$$

where

$$
\begin{aligned}
q & =2\left(\rho_{2} V_{S 2}^{2}-\rho_{1} V_{S 1}^{2}\right), \\
X & =\rho_{2}-q p^{2} \\
Y & =\rho_{1}+q p^{2} \\
Z & =\rho_{2}-\rho_{1}-q p^{2} \\
P_{1} & =\sqrt{1-V_{P 1}^{2} p^{2}} \\
Q_{1} & =\sqrt{1-V_{S 1}^{2} p^{2}} \\
P_{2} & =\sqrt{1-V_{P 2}^{2} p^{2}} \text { and } \\
Q_{2} & =\sqrt{1-V_{S 2}^{2} p^{2}} .
\end{aligned}
$$

In the form as above, the information about the angle of incidence is introduced into the reflection coefficients by the ray parameter $p$, and the quantities $P_{1}, P_{2}, Q_{1}$ and $Q_{2}$ corresponding to the cosines of reflection and transmission angles, respectively. As mentioned in the beginning, the exact elastic reflection coefficients depend only on velocities and densities of the media under study. In practice, reflection coefficients are manipulated using in inverse methodologies (Yilmaz, 1987). Taking prestack recorded seismograms, the reflection coefficients are fitted to amplitudes and petrophysical parameters are estimated (Shuey, 1985). For geological interpretation, petrophysical parameters can help characterizing sedimentary layers possibly working as oil and gas reservoirs. To obtain such an indication, simple expressions for the reflection coefficients can be derived by applying different methodologies on exact formulas (Ursin \& Dahl, 1992; Wang, 1999). For instance, Ursin \& Dahl (1992) treated the exact formula for the reflection coefficients simulating an acquisition geometry where offset between source and receivers tends to zero. In this way, a second-order approach is introduced in the mathematical formulation. The reflection coefficients were simplified by Wang (1999), assuming a pseudo-approximation for the ray parameter on Eqs. (5) and (6), and expanding only selected terms. Wang (1999) then derived approximated expressions depending on nonlinear contributions of the elastic parameters.

Concerning the objectives of this work, we evaluated only the response of exact elastic and poroelastic reflection coefficients for a selected geologic model. In other words, we neglect the linearized expressions. In the next section, we present the steps for determination of exact isotropic poroelastic reflection coefficients.

\section{Reflection coefficients for poroelastic media}

The assumption of a biphasic medium, i.e., porous rocks partially or fully saturated with a fluid, can increase enormously the complexity of the exact expressions for reflection coefficients. Biot (1956) fully described the theory for wave propagation in a fluid-saturated porous solid and discussed the corresponding implications. Deresiewicz \& Skalak (1963) checked Biot's theory using energy considerations of an incident wave at an interface separating two isotropic poroelastic layers. As a whole, the mathematical formulation of reflection coefficients in poroelastic media is similar to the elastic case. However, more two boundary conditions must be incorporated in poroelastic media. One additional boundary condition guarantees the continuity of the pressure field $P^{f}$; the other explains the relative displacement between solid and fluid $w^{z}$ along the direction normal to the interface. The set of six boundary conditions are then written as:
1. $u_{1}^{\prime z}=u_{2}^{\prime z}$,
2. $u_{1}^{\prime x}=u_{2}^{\prime}$,
3. $\tau_{1}^{\prime} z z=\tau_{2}^{\prime} z z$,
4. $\tau_{1}^{\prime} z x=\tau_{2}^{\prime z x}$,
5. $w_{1}^{z}=w_{2}^{z}$ and
6. $P_{1}^{f}=P_{2}^{f}$.

The boundary conditions 5 and 6 are introduced in the poroelastic reflection coefficient problem as a consequence of assuming a biphasic medium. By applying the preceding boundary conditions, it is possible to derive six coefficients instead of four as discussed in the elastic case. The additional two coefficients 
correspond to small-velocity transmitted and reflected compressional waves, that is, waves with velocity smaller than the conventional P-wave. As a result, a new terminology is used: Biot's wave or simply slow P-wave. The slow P-wave is predicted by the theory and is recorded using an acquisition geometry for characteristic frequencies of investigation.

Using plane-wave solutions to the particle displacements analogous to the in elastic case, and now for the relative displacement between solid and fluid $w$, the boundary conditions 1, 2 and 5 incorporate the slow P-wave into the reflection and transmission problem in poroelastic media. For the upper medium, $u_{1}^{\prime l}$ and $w_{1}^{z}$ are written as:

$$
\begin{gathered}
u_{1}^{\prime l}=A^{\prime l} \exp [i w(\mathbf{p} \cdot \mathbf{x}-t)] \\
+\sum_{j=1}^{3} A_{l}^{\prime} R_{j}^{\prime} \exp [i w(\mathbf{p} \cdot \mathbf{x}-t)]
\end{gathered}
$$

and

$$
\begin{gathered}
w_{1}^{z}=B^{\prime z} \exp [i w(\mathbf{p} \cdot \mathbf{x}-t)] \\
+\sum_{j=1}^{3} B^{\prime z} R_{j}^{\prime} \exp [i w(\mathbf{p} \cdot \mathbf{x}-t)] .
\end{gathered}
$$

For lower medium, $u_{l}^{\prime} b$ and $w_{z}^{b}$ are written as:

$$
u_{2}^{\prime l}=\sum_{j=1}^{3} A^{\prime l} T_{j}^{\prime} \exp [i w(\mathbf{p} \cdot \mathbf{x}-t)]
$$

and

$$
w_{2}^{z}=\sum_{j=1}^{3} B^{\prime z} T_{j}^{\prime} \exp [i w(\mathbf{p} \cdot \mathbf{x}-t)],
$$

where $l=x, z$. At the separating interface of both media, the continuity of stresses in the boundary conditions 3 and 4 can be estimated using the generalized Hooke's law for poroelastic media (Biot, 1962):

$$
\tau_{k l}=\left[(H-2 \mu) e_{j j}-C \xi\right] \delta_{k l}+2 \mu e_{k l} .
$$

The constitutive relation in the preceding equation requires a greater number of physical constants to describe soils under load if compared with stress-strain relation used to modeling elastic rocks - see Eq. (3). The constants introduced on poroelastic theory are required mainly by the effects of pore-pressure and fluid saturation. In this case, normal stresses $\left(\delta_{k l}=1\right.$ for $k=l$ ) introduce an additional contribution to the problem under analysis through the poroelastic constants $H, C, M$ and $\sigma$, evaluated as follows (Biot, 1962):

$$
\begin{gathered}
H=K_{\text {sat }}+\frac{4}{3} \mu+\sigma C, \\
C=\sigma M, \\
M=\frac{K_{g} K_{f}}{K_{g} \phi+K_{f}(\sigma-\phi)}
\end{gathered}
$$

and

$$
\sigma=1-\frac{K_{m a}}{K_{g}} .
$$

Noticeable is the direct dependence of the preceding poroelastic constants on the bulk moduli of the rock matrix $K_{m a}$, on the fluid saturation $K_{f}$, on the predominant solid grains $K_{g}$, on the porosity $\phi$, and on the shear modulus $\mu$. The term $K_{\text {sat }}$ stands for saturated bulk modulus of the rock.

The boundary condition 6 concerns the continuity of the pressure field. As shown in Biot (1956), the pressure field $P$ depends on the poroelastic constants related in Eqs. (14-17). As expected, the pressure field also depends on the solid dilatation $e_{j j}$, that is:

$$
P=M \xi-C e_{j j} .
$$

where $\xi=\phi \nabla . w$, for $w$ defined as the difference between the solid and fluid particle displacements - see in Eq. (A1) of the Appendix.

This brief discussion about how to expand the boundary conditions proposed by Deresiewicz \& Skalak (1963) provides the derivation of a linear system of algebraic equations to determinate the reflection and transmission coefficients in poroelastic media. Equations (9-12) form the continuity conditions for solid grains displacement and relative displacement between solid and fluid. The relations for the continuity of stresses and the pressure field are established by Eqs. (13) and (18) in addition to Eqs. (9-12). As a result, the linear system of six algebraic equations can be expressed in matrix form as:

$$
\overline{\mathbf{A}}_{m n} \overline{\mathbf{X}}_{n}=\overline{\mathbf{Y}}_{m} .
$$

That is: the coefficients matrix $\overline{\mathbf{X}}_{n}$ has six terms corresponding to three reflected and three transmitted waves. The additional two coefficients in comparison with elastic case - see Eq. (4) - correspond to reflection and transmission coefficients of the slow P-wave.

Unfortunately, due to the amount of constants involved in the poroelastic case, a treatable analytical solution for reflection and transmission coefficients is impossible to derive. In the next section, we generate the coefficient numerically, by calculating 
matrix $\overline{\mathbf{X}}_{n}$ in Eq. (19). Note that only the fast P-wave reflection coefficient $\left(R_{P P}^{\text {poro }}\right)$ and the converted shear-wave reflection coefficient $\left(R_{P S}^{\text {poro }}\right)$ in poroelastic media are considered. The superscript "poro" emphasizes the approximation for the media forming the geological model under consideration, namely, formed by homogeneous isotropic poroelastic media.

\section{RESULTS}

In this section, we numerically investigate the parameter dependence of the exact reflection coefficients in homogeneous isotropic poroelastic media. We focus on the parameter dependence of the generated fast $\mathrm{P}$-wave $\left(R_{P P}^{\text {poro }}\right)$ and the converted S-wave $\left(R_{P S}^{\text {poro }}\right)$ reflection coefficients. As mentioned above, the geological model consists of a discontinuity interface separating two homogeneous isotropic poroelastic media. A fast incident Pwave is assumed to impinge on the discontinuity interface from the upper poroelastic layer. The upper layer represents a clastic reservoir rock, while the lower sealing layer simulates a carbonate rock. The fluid saturation was considered as water in both layers to simulate the continuity of reservoir. The pores were assumed fully saturated to evidence the effects of fluid saturation and pore pressure. Although has considered water as fluid saturation, this study also can be applied for hydrocarbon in view of theoretical response of poroelasticity. The basic values for the physical properties of dry rocks (i.e., bulk density $\rho_{m a}$ and incompressibility $K_{g}$ of the rock matrix, and the velocities $V_{P}$ and $V_{S}$ ) are shown in Table 1. These values are relevant for calculation of the poroelastic constants defined in the poroelastic theory (Biot, 1956; Gassmann, 1951; Wyllie et al., 1958) - see the Appendix

In order to evaluate the poroelastic reflection coefficients $R_{P P}^{\text {poro }}$ and $R_{P S}^{\text {poro }}$ at the interface of the proposed geological model, we considered the reservoir porosity (i.e., $\phi_{1}$ for the upper layer), the frequency content of the seismic source $f$ and the incidence angle $\theta$ as the main parameters of the dependence. We retained the porosity constant for the lower layer (i.e., $\phi_{2}=5 \%$ ), as long as it simulated a sealing carbonate rock. Then, we provide numerical calculation of $R_{P P}^{\text {poro }}$ and $R_{P S}^{\text {poro }}$ using the following range of variation for the dependence parameters: $5 \% \leq \phi_{1} \leq$ $30 \%, 0^{\circ} \leq \theta \leq 40^{\circ}$ and $10 \mathrm{~Hz} \leq f \leq 200 \mathrm{~Hz}$. By assuming the indicence angle $\theta$ as a dependence parameter, we envisage indirectly studying the influence of the source-to-receiver distances (i.e., of the offsets used in acquisition geometries for seismic surveys) on poroelastic reflection amplitudes. We further represent the poroelastic reflection coefficients using 3D plots. The coefficients are displayed along the vertical axis, as the two other horizontal axes show specific parameters of the dependence.
Figure 1 shows how the coefficients $R_{P P}^{\text {poro }}$ vary depending on the reservoir porosity $\phi_{1}$ and incidence angle $\theta$. In Figure 2, similar dependence parameters are selected to represent the absolute values of the converted coefficients $R_{P S}^{\text {poro }}$. The choice for representing the absolute values of the coefficients is explained because we aimed only at studying the variation of their magnitudes. In both figures, calculations of reflection coefficients was taken assuming the frequency of investigation as $f=100 \mathrm{~Hz}$. Figure 1 reveals that, for lower reservoir porosities $\phi_{1}$ and incidences near to zero-offset $\left(\theta=0^{\circ}\right)$, the magnitudes of $R_{P P}^{\text {poro }}$ coefficient reach higher values. The $R_{P P}^{\text {poro }}$ coefficients decrease magnitudes as offset and reservoir porosity increase. Nevertheless, in respect to the variation of the $R_{P P}^{\text {poro }}$ coefficients, Figure 2 shows an opposite behaviour for the $R_{P S}^{\text {poro }}$ coefficients. That is: the higher magnitudes for the $R_{P S}^{\text {poro }}$ coefficients occur as the incidence angle increases and for small values of the reservoir porosity $\phi_{1}$. In addition, increasing the reservoir porosity $\phi_{1}$ leads to a decrease of the magnitudes of the $R_{P S}^{\text {poro }}$ coefficients. This effect can be interpreted as a consequence of assuming 100\% saturation for the reservoir rock.

In order to compare the magnitudes of reflection coefficients in elastic and poroelastic media, we calculated the corresponding isotropic elastic coefficients (i.e., $R_{P P}^{\text {elast }}$ and $R_{P S}^{\text {elast }}$ ) using Eqs. (5) and (6). The reservoir porosity $\phi_{1}$ and incidence angle $\theta$ were also assumed as the same dependence parameters. For each layer of the geologic model, bulk densities $\rho_{b}$ and velocities $V_{P}$ and $V_{S}$ were defined as the same values applied in the poroelastic experiment. Figures 3 and 4 show the absolute residuals $\left|R_{P P}^{\text {elast }}-R_{P P}^{\text {poro }}\right|$ and $\left|R_{P S}^{\text {elast }}-R_{P S}^{\text {poro }}\right|$, respectively. The behaviour of these absolute residuals shows significance only for specific situations. For reservoir porosities below $5 \%$ and incidence angles above $15^{\circ}$, the absolute difference $\left|R_{P P}^{\text {elast }}-R_{P P}^{\text {poro }}\right|$ is higher than 0.5. The maximum absolute difference value is 1.3 (see Fig. 3). In turn, the absolute difference for the converted S-wave coefficients $\left|R_{P S}^{\text {elast }}-R_{P S}^{\text {poro }}\right|$ in Figure 4 hardly reaches 0.5 , a magnitude which corresponds to intermediate incidence angles (i.e., $15^{\circ}<\theta<25^{\circ}$ ) and low reservoir porosities nearly below $8 \%$. As a result, classical AVO analysis using the converted S-wave isotropic reflection coefficient approximation can be concentrated at this region of incidence.

Besides the very distinct parameter dependence in comparison to elastic coefficients, the poroelastic reflection coefficients also vary with frequency $f$. This represent a crucial factor in order to obtain a good vertical resolution and to illuminate the geological objectives defined beforehand (Yilmaz, 2001; Rosa, 2010). 
Table 1 - Upper and lower empty rock matrix properties. Velocities of dry rocky frame $V_{P}$ and $V_{S}$ in $(\mathrm{m} / \mathrm{s})$, density $\rho_{m a}$ in $\mathrm{kg} / \mathrm{m}^{3}$, bulk modulus of composed grains $K_{g}$ in $(\mathrm{GPa})$ and permability coefficient $k$ in $\left(\mathrm{m}^{2}\right)$. For the fluid (water) saturated porous reservoir, the physical properties are: $10^{3} \mathrm{~kg} / \mathrm{m}^{3}$ for density $\rho_{f}, 2.4 \mathrm{GPa}$ for incompressibility $K_{f}$, and $10^{-2}$ Poise for absolute viscosity $\eta$.

\begin{tabular}{|c|c|c|c|c|c|}
\hline Properties & $V_{P}(\mathrm{~m} / \mathrm{s})$ & $V_{S}(\mathrm{~m} / \mathrm{s})$ & $\rho_{m a}\left(\mathrm{~kg} / \mathrm{m}^{3}\right)$ & $K_{g}(\mathrm{GPa})$ & $k\left(\mathrm{~m}^{2}\right)$ \\
\hline upper & 3000 & 1800 & 2650 & 30 & $10^{-9}$ \\
lower & 3500 & 2000 & 2750 & 50 & $10^{-12}$ \\
\hline
\end{tabular}

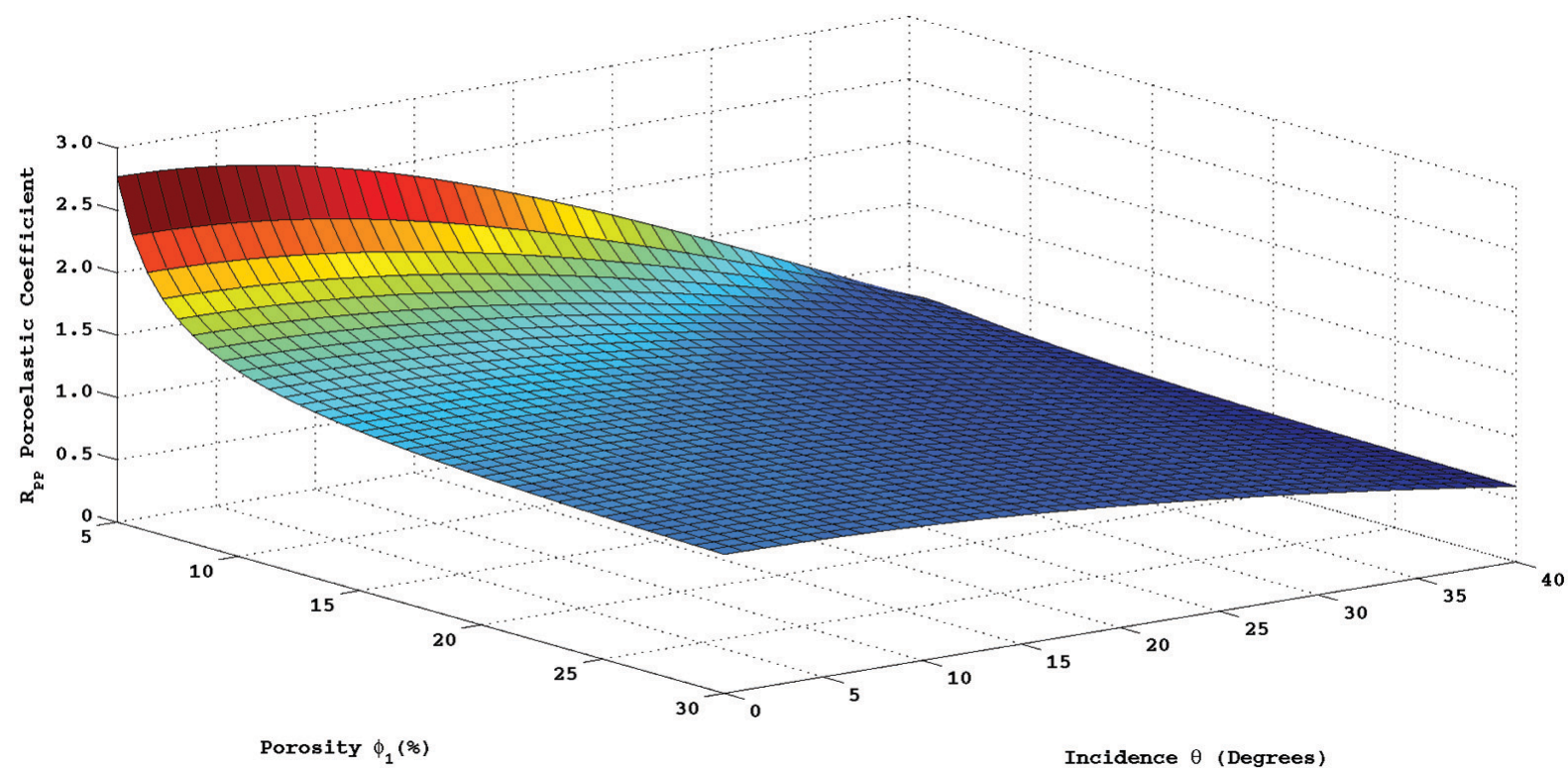

Figure 1 - Fast P-wave $R_{P P}^{\text {poro }}$ reflection coefficients in homogeneous isotropic poroelastic media as a function of the incidence angle $\theta$ and the reservoir porosity $\phi_{1}$, that is, $R_{P P}^{\text {poro }}\left(\theta, \phi_{1}\right)$. The frequency investigation is assumed constant $f=100 \mathrm{~Hz}$.

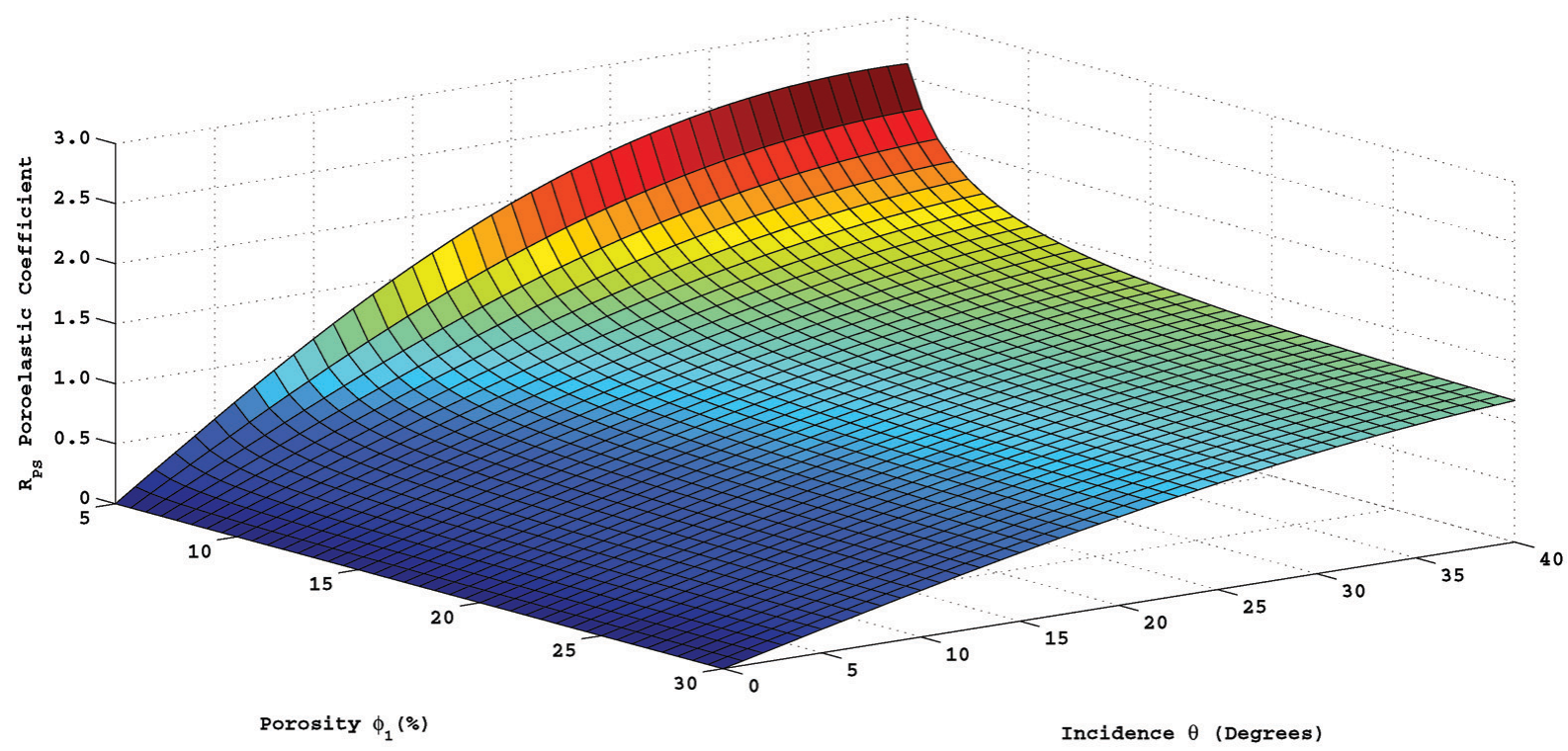

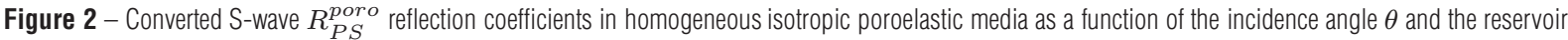
porosity $\phi_{1}$, that is, $R_{P S}^{\text {poro }}\left(\theta, \phi_{1}\right)$. The frequency investigation is assumed constant $f=100 \mathrm{~Hz}$. 


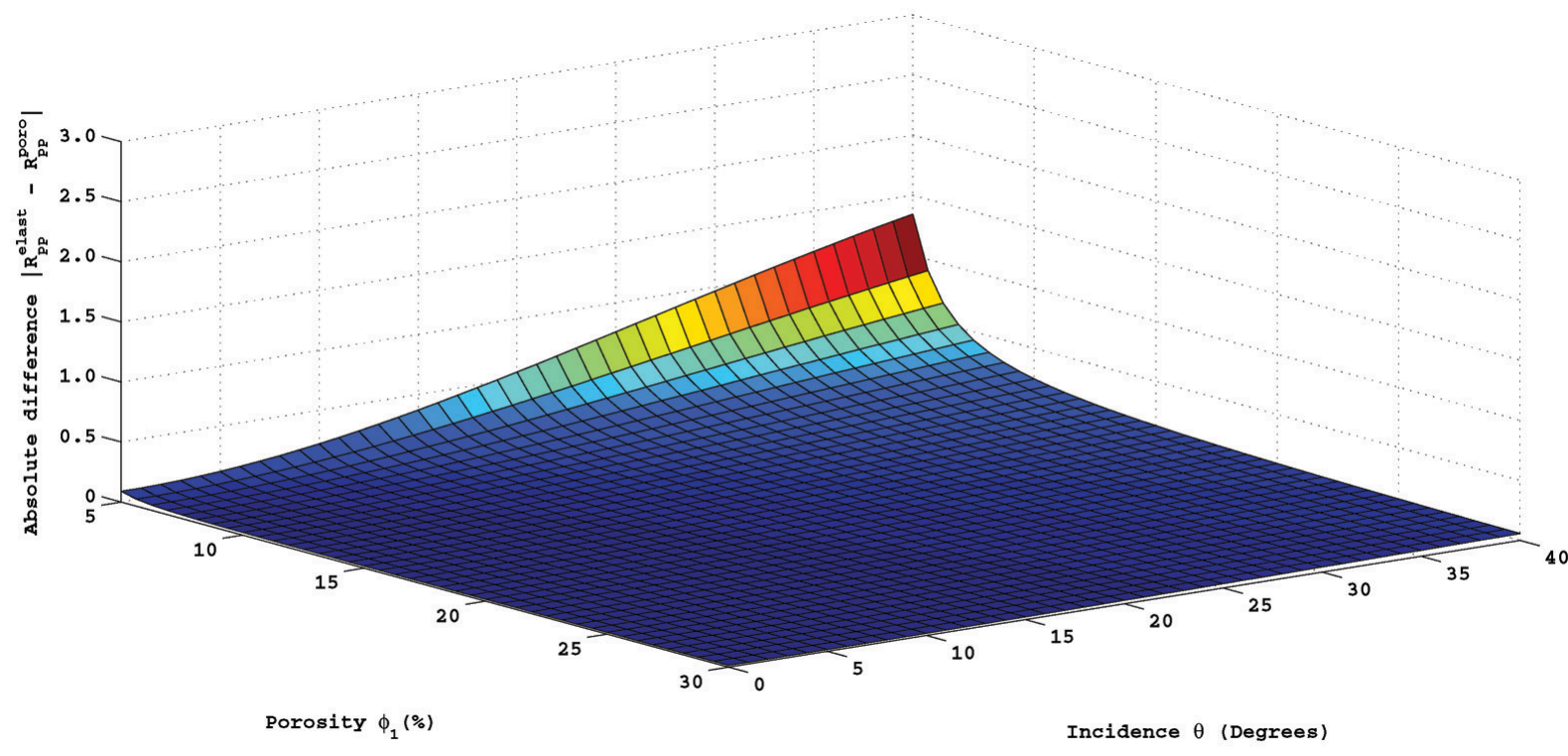

Figure 3 - Absolute differences between the P-wave reflection coefficients in elastic media and the fast P-wave reflection coefficients in poroelastic media, i.e. $\left|R_{P P}^{\text {elast }}-R_{P P}^{\text {poro }}\right|$. The poroelastic coefficients $R_{P P}^{\text {poro }}$ were calculated for a frequency $f=100 \mathrm{~Hz}$. Calculations of $\left|R_{P P}^{\text {elast }}-R_{P P}^{\text {poro }}\right|$ coefficients took into account the dependence on the incidence angle $\theta$ and reservoir porosity $\phi_{1}$. The geological model is described in the text.

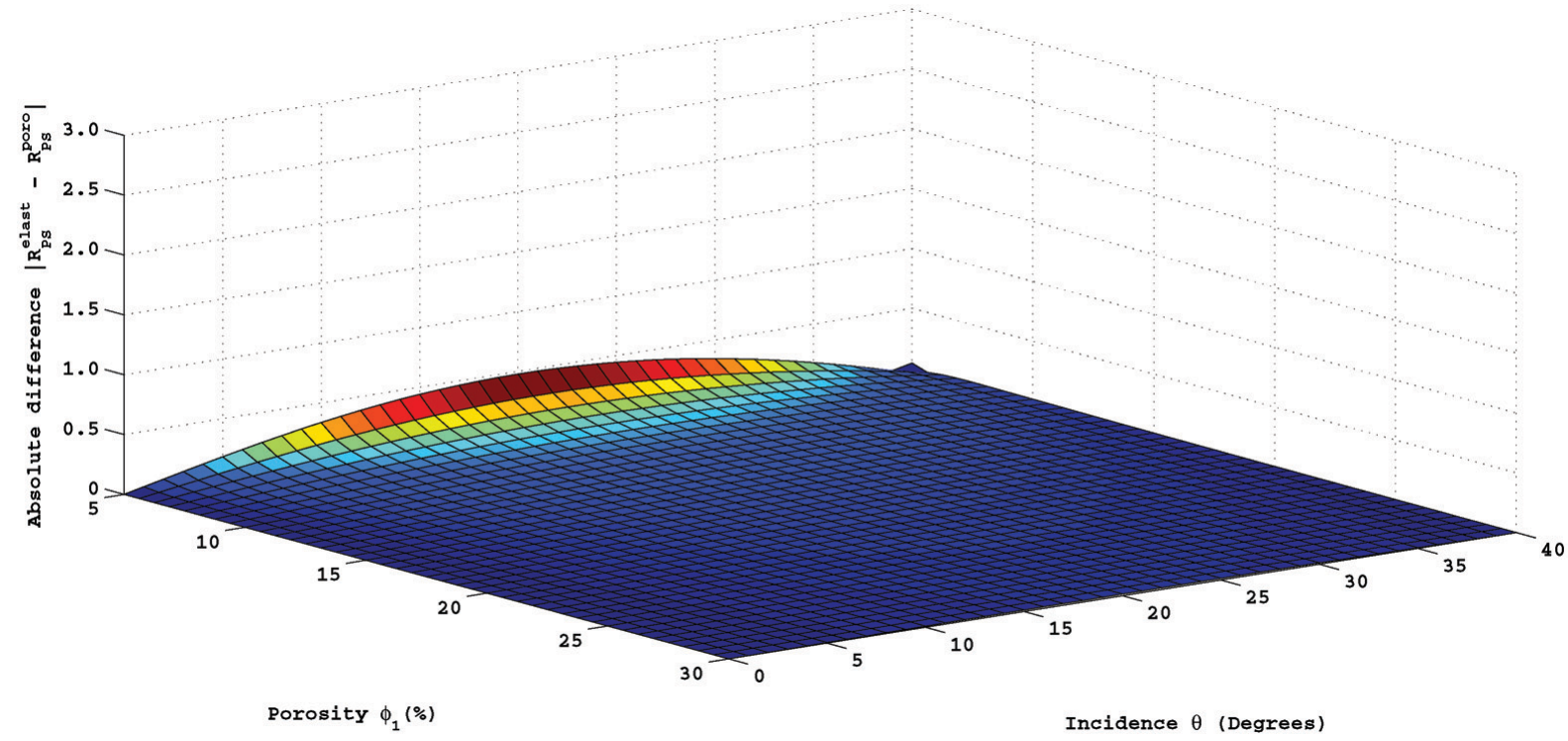

Figure 4 - Absolute differences between the converted S-wave reflection coefficients in elastic media and the converted S-wave reflection coefficients in poroelastic media, i.e., $\left|R_{P S}^{\text {elast }}-R_{P S}^{\text {poro }}\right|$. The poroelastic coefficients $R_{P S}^{\text {poro }}$ were calculated for a frequency $f=100 \mathrm{~Hz}$. Calculations of $\left|R_{P S}^{\text {elast }}-R_{P S}^{\text {poro }}\right|$ coefficients took into account the dependence on the incidence angle $\theta$ and reservoir porosity $\phi_{1}$. The geological model is described in the text.

Thus, we also calculated the variations of the poroelastic coefficients $R_{P P}^{\text {poro }}$ and $R_{P S}^{\text {poro }}$ varying the reservoir porosity $\phi_{1}$ and frequency $f$. However, for interpreting the results, we selected a constant value for the incidence angle, namely, $\theta=15^{\circ}$. The results of the calculations are plotted in Figures 5 and 6 , respec- tively, for the poroelastic $R_{P P}^{\text {poro }}$ and $R_{P S}^{\text {poro }}$ coefficients. By inspecting both plots, it can be seen that the poroelastic $R_{P P}^{\text {poro }}$ and $R_{P S}^{\text {poro }}$ coefficients hold weak dependence on frequency. Once again, as the reservoir porosity varies, fluid saturation is the dominant factor affecting the coefficients. 


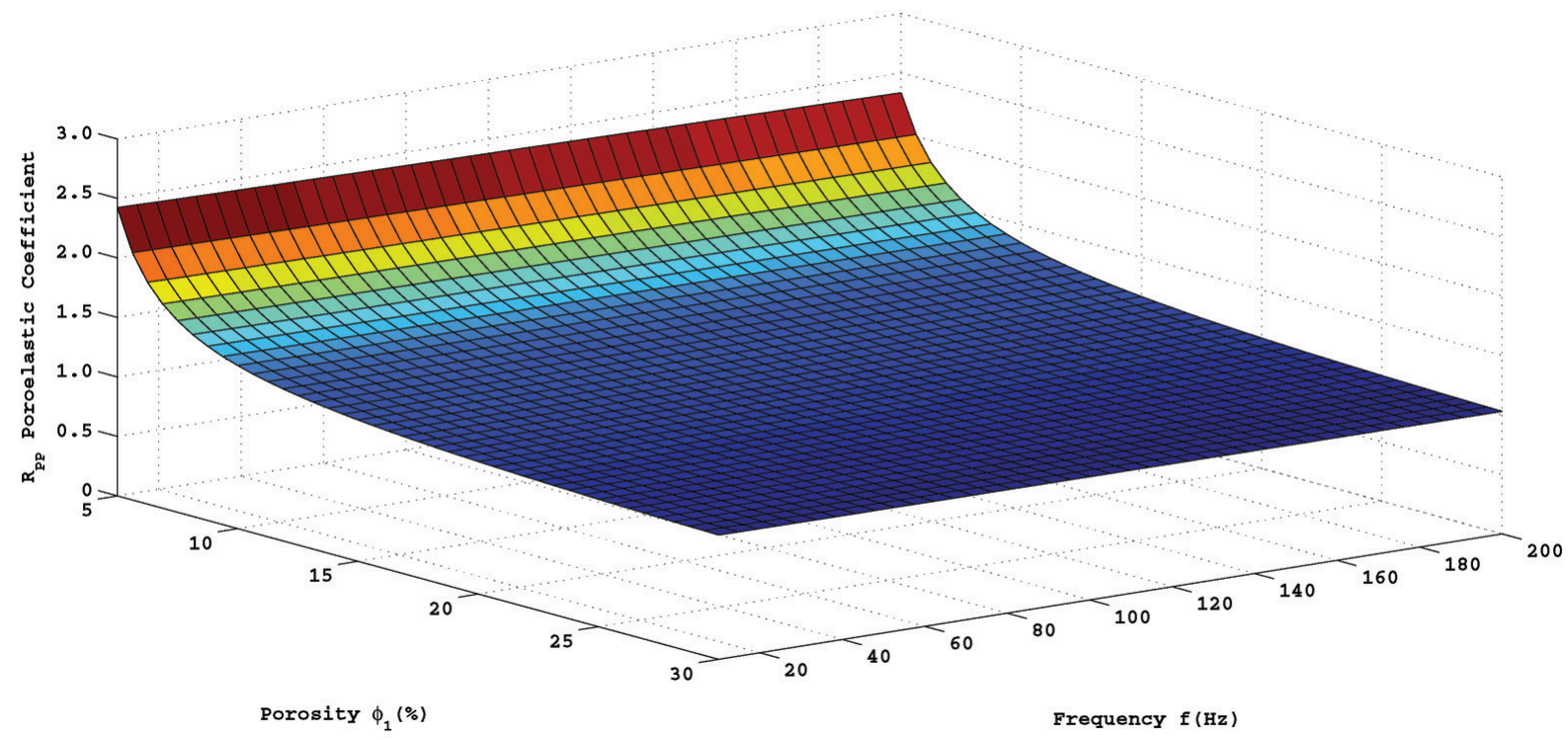

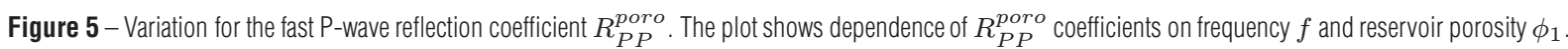
An intermediate incidence angle is assumed in the calculations (i.e., $\theta=15^{\circ}$ ). The geologic model is described in the text.

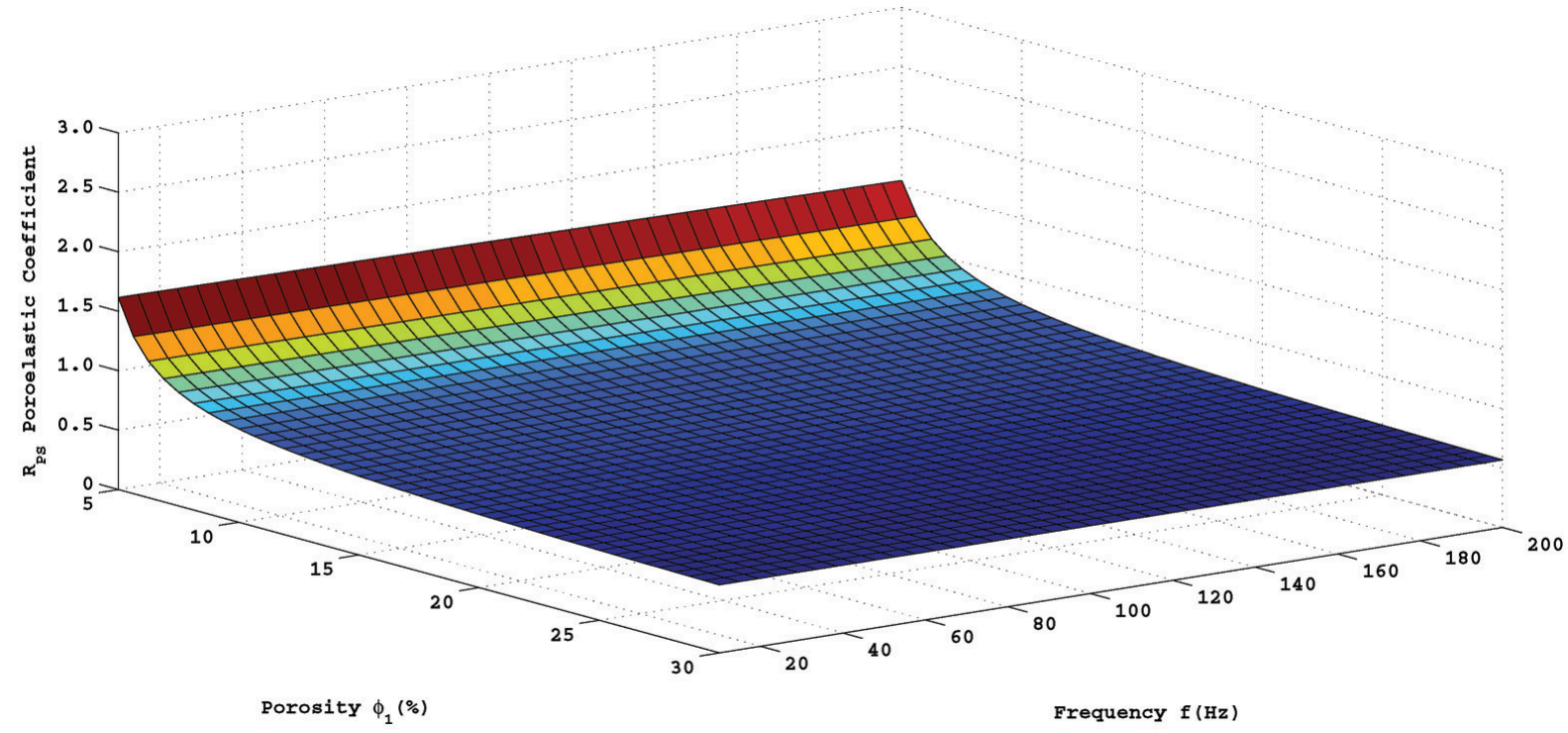

Figure 6 - Variation for the converted S-wave reflection coefficient $R_{P S}^{\text {poro }}$. The plot shows dependence of $R_{P S}^{\text {poro }}$ coefficients on frequency $f$ and reservoir porosity $\phi_{1}$. An intermediate incidence angle is assumed in the calculations (i.e., $\theta=15^{\circ}$ ). The geologic model is described in the text.

Also for comparison purposes, we calculated the absolute differences between elastic and poroelastic P-wave and converted S-wave reflection coefficients as $\left|R_{P P}^{\text {elast }}-R_{P P}^{\text {poro }}\right|$ and $\left|R_{P S}^{\text {elast }}-R_{P S}^{\text {poro }}\right|$. Now, the dependence is on the reservoir porosity $\phi_{1}$ and frequency $f$. The results are plotted in Figures 7 and 8, respectively, for $\left|R_{P P}^{\text {elast }}-R_{P P}^{\text {poro }}\right|$ and $\left|R_{P S}^{\text {elast }}-R_{P S}^{\text {poro }}\right|$. In both situations, the influence of frequency $f$ is remarkably insignificant. This is an indication that, at least for the range used here, the frequency of investigation hardly promotes large differences in the magnitudes of elastic and poroelastic reflection coefficients. For frequencies investigations of the order of ultrasonic frequencies the obtained results between elastic and poroelastic backgrounds is noticeably different as shown in literature (Gurevich et al., 2004). However for purposes of conventional seismic acquisition are not used the frequencies of investigation of the order of $\mathrm{kHz}$ (Yilmaz, 2001; 


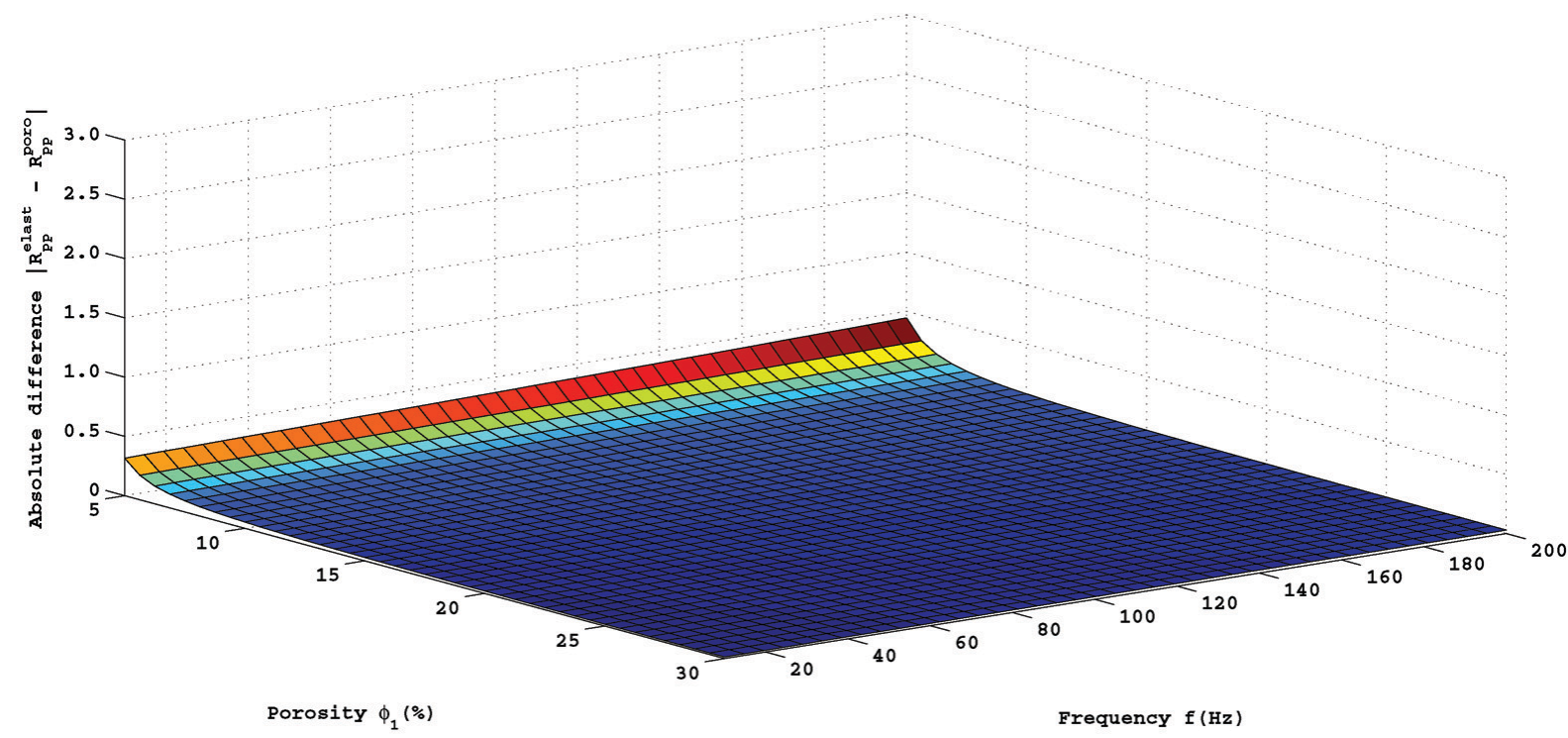

Figure 7 - Absolute differences between the elastic and poroelastic P-wave reflection coefficients, i.e., $\left|R_{P P}^{\text {elast }}-R_{P P}^{\text {poro }}\right|$. The magnitudes of the absolute differences vary with the frequency $f$ and the reservoir porosity $\phi_{1}$. An intermediate incidence angle $\theta=15^{\circ}$ is assumed in the calculations. The geologic model is discussed in the text.

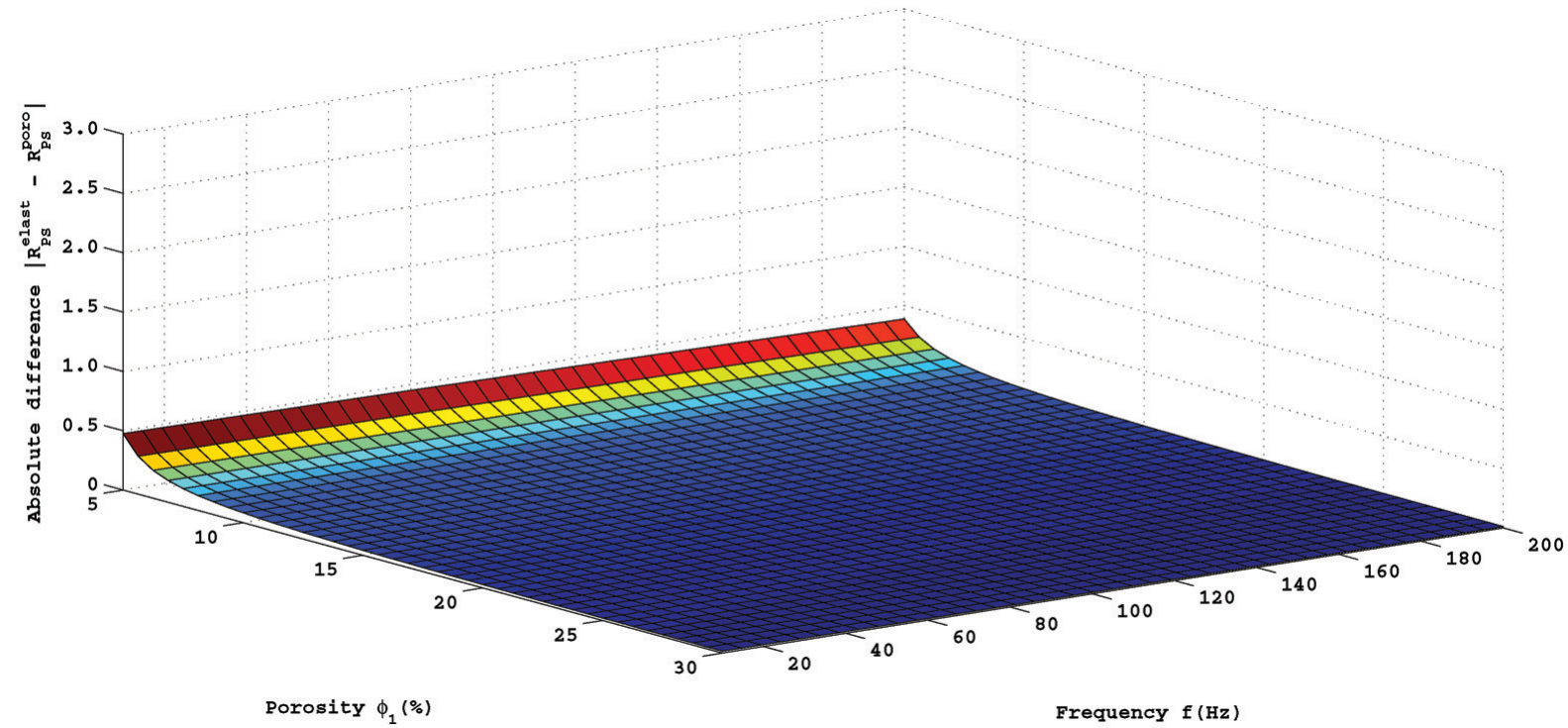

Figure 8 - Absolute differences between the elastic and poroelastic converted S-wave reflection coefficients, i.e., $\left|R_{P S}^{\text {elast }}-R_{P S}^{\text {poro }}\right|$. The magnitudes of the absolute differences vary with the frequency $f$ and the reservoir porosity $\phi_{1}$. An intermediate incidence angle $\theta=15^{\circ}$ is assumed in the calculations. The geologic model is discussed in the text.

Rosa, 2010). In order to guarantee an even solid analysis, we present in Table 2 simple statistical measures.

\section{DISCUSSION AND CONCLUSIONS}

The AVO technique represent a valuable tool for characterization of reflection amplitude anomalies. The development of oil and gas reservoirs can benefit from the estimatives of the petrophysical properties so obtained. In the context of classic AVO analysis, seismic amplitude data are fitted to linearized expressions of isotropic elastic reflection coefficients. Nevertheless, fluidsaturated porous rocks are better approximated by the poroelastic theory, for which linearized expressions for reflection coeffi- 
Table 2 - Statistics for uncertainty analysis of the magnitudes of poroelastic reflection coefficients of $R_{P P}$ and $R_{P S}$, as well as the absolute differences $\left(R_{P P}^{\text {elast }}-R_{P P}^{\text {poro }}\right)$ and $\left(R_{P S}^{\text {elast }}-R_{P S}^{\text {poro }}\right)$ in Figures 1 to 4 , on dependence of incidence angle $\theta$ and porosity $\phi_{1}$. (b) Statistics for uncertainty analysis of the magnitudes of poroelastic reflection coefficients of $R_{P P}$ and $R_{P S}$, as well as the absolute differences $\left(R_{P P}^{\text {elast }}-R_{P P}^{\text {poro }}\right)$ and $\left(R_{P S}^{\text {elast }}-R_{P S}^{\text {poro }}\right)$ in Figures 5 to 8 , on dependence of frequency $f$ and porosity $\phi^{1}$. Computation of minimum, maximum and mean values, and standard deviation $\sigma$ and variance $\sigma^{2}$.

\begin{tabular}{|c|c|c|c|c|}
\hline$(\mathrm{a})$ & $R_{P P}\left(\theta, \phi_{1}\right)$ & $R_{P S}\left(\theta, \phi_{1}\right)$ & $\left|R_{P P}^{\text {elast }}-R_{P P}^{\text {poro }}\right|$ & $\left|R_{P S}^{\text {elast }}-R_{P S}^{\text {poro }}\right|$ \\
\hline $\min$ & 0.6054 & 0.0000 & 0.0148 & 0.0000 \\
$\max$ & 2.7673 & 2.6156 & 1.3415 & 0.4781 \\
$\operatorname{mean}$ & 1.1109 & 0.8427 & 0.0955 & 0.0459 \\
$\sigma$ & 0.3335 & 0.4976 & 0.1483 & 0.0684 \\
$\sigma^{2}$ & 0.1112 & 0.2476 & 0.0220 & 0.0047 \\
\hline \hline$(\mathrm{b})$ & $R_{P P}\left(\mathrm{f}, \phi_{1}\right)$ & $R_{P S}\left(\mathfrak{f}, \phi_{1}\right)$ & $\left|R_{P P}^{\text {elast }}-R_{P P}^{\text {poro }}\right|$ & $\left|R_{P S}^{\text {elast }}-R_{P S}^{\text {poro }}\right|$ \\
\hline $\min$ & 1.0301 & 0.5691 & 0.0137 & 0.0119 \\
$\max$ & 2.4229 & 1.6152 & 0.4449 & 0.4761 \\
$\operatorname{mean}$ & 1.2497 & 0.7165 & 0.0601 & 0.0635 \\
$\sigma$ & 0.2885 & 0.2104 & 0.0680 & 0.0836 \\
$\sigma^{2}$ & 0.0832 & 0.0442 & 0.0046 & 0.0070 \\
\hline
\end{tabular}

cients are unavailable. As a result, studies on how poroelastic reflection coefficients varies with petrophysical parameters must be accomplished numerically.

In this paper, we numerically investigated the variation of isotropic poroelastic reflection coefficients for the fast $\mathrm{P}$-wave and converted S-wave, respectively, $R_{P P}^{\text {poro }}$ and $R_{P S}^{\text {poro }}$. The geologic model consisted of a discontinuity interface separating two poroelastic layers. The upper layer simulates a water-saturated clastic reservoir, while the lower layer represents a sealing carbonate rock with constant porosity (i.e., $\phi_{2}=5 \%$ ). An incident fast $P$-wave impinges the interface from above at a given angle of incidence $\theta$. In order to represent the poroelastic reflection coefficients, we used $3 \mathrm{D}$ plots in which the vertical axis displays the coefficients. The two other horizontal axes show specific parameters of the dependence, that is: the reservoir porosity $\phi_{1}$ and the incidence angle $\theta$, or the reservoir porosity $\phi_{1}$ and the dominant frequency $f$. The dependence of the coefficients on frequency is an implication from the poroelastic theory. Still, using the incidence angle $\theta$ as a main parameter of the dependence, we indirectly analyzed the influence of seismic acquisition offsets on the reflection amplitudes. Consequently, two fundamental conclusions can be drawn from the numerical poroelastic reflection coefficients presented in Figures 1-8. First, reflection amplitudes in poroelastic layers have weak dependence on frequency $f$, at least considering the range used for calculations (i.e., $10 \mathrm{~Hz} \leq f \leq 200 \mathrm{~Hz}$ ). Secondly, it can be observed a strong dependence of reflection coefficients on medium porosity; as shown in the results, such a dependence is even higher for fluid-filled pores. Still considering porosity, we call attention to the similarities between reflection coefficients in elastic and poroelastic media.

\section{ACKNOWLEDGMENTS}

This paper is product of the project "Caracterização de Anisotropia Sísmica Usando Perfis Geofísicos de Poços de Petróleo e Gás", supported by CNPq/Brazil - the Brazilian Agency for the Development of Science \& Technology (proc. 471647/2006-3). José Oliveira acknowledges the scholarship from CAPES/Brazil, supporting the development of his doctoral thesis in the PostGraduation Course in Geophysics at Observatório Nacional/ Brazil. Jorge L. Martins acknowledges a research grant from CNPq/Brazil (proc. 302480/2011-0).

\section{APPENDIX}

A brief summary of the theory of wave propagation in poroelastic medium is presented below. Details can be found in Biot (1956). In the low-frequency range, the equations for phase velocities in a poroelastic medium can be derived by starting with the system of coupled equations (Biot, 1956):

$$
\begin{gathered}
\rho_{b} \frac{\partial^{2} u}{\partial t^{2}}+\rho_{f} \frac{\partial^{2} w}{\partial t^{2}} \\
=(\lambda+\mu) \nabla(\nabla . u)+\mu \nabla^{2} u+2 \sigma D \nabla(\nabla . w)
\end{gathered}
$$


and

$$
\begin{aligned}
& \rho_{f} \frac{\partial^{2} u}{\partial t^{2}}+m \frac{\partial^{2} w}{\partial t^{2}}+\frac{\eta}{k} \frac{\partial w}{\partial t} \\
= & 2 \sigma D \nabla(\nabla \cdot u)+2 D \nabla(\nabla \cdot w) .
\end{aligned}
$$

The above equations describe the propagation of existing waves in a poroelastic medium. It can be seen that such a propagation depends on particle displacement $u$, on mineral grains constituting the rock frame and on relative displacement $w$ between the solid and fluid phases of the saturated rock. The uncoupling of Eqs. (A1) and (A2) reveals the existence of three elastic waves: a shearing wave (i.e., with transversal mode of vibration), and two Iongitudinal waves (i.e., having vibration along the direction of propagation). One of these longitudinal waves corresponds to the classical P-wave. The other P-wave has lower velocity in comparison to this classical wave, and is defined as Biot's wave or slow P-wave.

The quantities presented in Eqs. (A1) and (A2) are discussed in several papers. For instance: the mass-coupled coefficient $m$, viscosity $\eta$ and permeability $k$. The effective density $\rho_{b}$ can be rewrite as a function of contributions of matrix grains $\rho_{m a}$ and fluid saturation $\rho_{f}$. For the cases in which the rock matrix can be considered monomineralogic, the effective density $\rho_{b}$ are determinated as follows (Wyllie et al., 1958):

$$
\rho_{b}=(1-\phi) \rho_{m a}+\phi \rho_{f},
$$

where $\phi$ is the total porosity of the fully saturated rocks. The terms $\sigma$ and $D$ are poroelastic constants, which are defined by the bulk moduli of rock matrix $K_{m a}$, of mineral grains $K_{g}$ and of fluid $K_{f}$. As $\sigma$ is related in Eq. (17), $D$ is expressed as follows:

$$
D=\frac{K_{g}}{2}\left[\sigma+\frac{\phi}{K_{f}}\left(K_{g}-K_{f}\right)\right]^{-1},
$$

where $\lambda$ and $\mu$ are Lamé's parameters. The shear modulus $\mu$ is defined as the same for dry or saturated rocks, as already presented in other works (Toksöz et al., 1976). This quantitiy can be estimated, in a simplified way, using the S-wave velocity of dry rocks. Similarly, determination of the bulk modulus of the rock matrix $K_{m a}$ can be accomplished using the P-wave velocity, and including the fluid saturation effects (Gassmann, 1951):

$$
K_{s a t}=K_{g}\left(\frac{K_{m a}+Q}{K_{g}+Q}\right),
$$

where

$$
Q=\frac{K_{f}}{\phi}\left(\frac{K_{g}-K_{m a}}{K_{g}-K_{f}}\right) .
$$

The resulting equations for calculating fast $\mathrm{P}$ - and $\mathrm{S}$-wave velocities $V_{P}$ and $V_{S}$ in poroelastic medium are (Biot, 1956):

$$
V_{P}=W \sqrt{\frac{4 \sigma^{2} D^{2}-2 D H}{\rho_{f}\left(H \sigma_{P}+2 \sigma D\right)}}-
$$

and

$$
V_{S}=W \sqrt{\frac{\mu}{\rho_{b}}\left(\frac{\rho_{b} \sigma_{S}}{\rho_{b} \sigma_{S}-\rho_{f}}\right)} .
$$

The quantities $\sigma_{P}$ and $\sigma_{S}$ arise from uncoupling the system of Eqs. (A1) and (A2):

$$
\begin{aligned}
\sigma_{P}=-( & H \rho_{f} k W+H i \eta \phi+2 D \rho_{b} \phi k W+\left(H^{2} \rho_{f}^{2} k^{2} W^{2}-2 H^{2} \rho_{f} k W i \eta \phi-4 H \rho_{f} k^{2} W^{2} D \rho_{b} \phi\right. \\
& +H^{2} i^{2} \eta^{2} \phi^{2}+4 H i \eta \phi^{2} D \rho_{b} k W+4 D^{2} \rho_{b}^{2} \phi^{2} k^{2} W^{2}-8 \phi k^{2} W^{2} H \rho_{f}^{2} \sigma D \\
& +8 \phi^{2} k W H \rho_{f} \sigma D i \eta+8 \phi^{2} k^{2} W^{2} H \rho_{f}^{2} D+16 \phi k^{2} W^{2} \sigma^{2} D^{2} \rho_{b} \rho_{f} \\
& \left.-16 \phi^{2} k W \sigma^{2} D^{2} \rho_{b} i \eta-16 \phi^{2} k^{2} W^{2} \sigma D^{2} \rho_{b} \rho_{f}\right)^{1 / 2} /\left(2 \phi k W\left(-H \rho_{f}+2 \sigma D \rho_{b}\right)\right),
\end{aligned}
$$

and

$$
\sigma_{S}=\frac{1}{\rho_{f}}\left(\frac{i \eta}{k W}-m\right)
$$

In the equations above, $W=2 \pi f$. In most published papers, we found the Eq. (A10) rather than the equation for $\sigma_{P}$. Here, we included Eq. (A9) after a mathematical treatment to Eqs. (A1) and (A2) using a algebraic manipulation software. 


\section{REFERENCES}

AKI K \& RICHARDS PG. 1980. Quantitative Seismology - Theory and Methods. W.H. Freeman \& Co., Vol. I, Chapter 5: 153.

BIOT MA. 1956. Theory of propagation of elastic waves in a fluidsaturated porous solid. Acoust. Soc. of Am., 28(2): 168-191.

BIOT MA. 1962. Mechanics of deformation and acoustic propagation in porous media. J. of Appl. Phys., 33: 1482-1498.

BORTFELD R. 1961. Approximation to the reflection and transmission coefficients of plane longitudinal and transverse waves. Geophys. Prosp., 9: 485-502.

DENNEMAN AIM, DRIJKONINGEN GG, SMEULDERS DMJ \& WAPENAAR K. 2002. Reflection and transmission of waves at a fluid/ porous $-m$ medium interface. Geophysics, 67: 282-291.

DERESIEWICZ H \& SKALAK R. 1963. On uniqueness in dynamic poroelasticity. Bulletin of the Seism. Society of America, 53: 409-416.

GASSMANN F. 1951. Elasticity of porous media. Vierteljahrsschr der Naturforschenden Gesselschaft, 96: 1-23.

GUREVICH B, CIZ R \& DENNEMAN AIM. 2004. Simple expressions for normal incidence reflection coefficients from an interface between fluid-saturated porous materials. Geophysics, 69: 1372-1377.

OSTRANDER WJ. 1984. Plane-wave reflection coefficients for gas sands at nonnormal angles of incidence. Geophysics, 49(10): 1637-1648.

QUINTAL B, STEFAN MS \& PODLADCHIKOV YY. 2011. Impact of fluid saturation on the reflection coefficient of a poroelastic layer. Geophysics, 76: $1-12$.

ROSA ALR. 2010. Análise do Sinal Sísmico. Sociedade Brasileira de Geofísica. Rio de Janeiro, Brazil. (/n Portuguese). 668 pp.

SHARMA MD. 2008. Wave propagation across the boundary between two dissimilar poroelastic solids. Journal of Sound and Vibration, 314: 657-671.
SHUEY RT. 1985. A simplification of the Zoeppritz equations. Geophysics, 50: 609-614.

URSIN B \& DAHL T. 1992. Seismic reflection amplitudes. Geophysical Prospecting, 40: 483-512.

URSIN B \& TJÄLAND E. 1996. The information content of the elastic reflection matrix. Geophys. J. Int., 125: 214-228.

THOMSEN L. 1986. Weak Elastic Anisotropy. Geophysics, 51(10): 1954-1966.

TOKSÖZ M, CHENG C \& TIMUR A. 1976. Velocities of seismic waves in porous rocks. Geophysics, 41: 621-645.

VAVRYČUK V \& PŠENČíK I. 1998. PP-wave reflection coefficients in weakly anisotropic media. Geophysics, 63: 2129-2141.

WANG Y. 1999. Approximations to the Zoeppritz equations and their use in AVO analysis. Geophysics, 64: 1920-1927.

WHITE JE. 1975. Computed seismic speeds and attenuation in rocks with partial gas saturation. Geophysics, 40: 224-232.

WYLLIE MRJ, GREGORY AR \& GARDNER LW. 1958. An experimental investigation of factors affecting elastic wave velocities in porous media. Geophysics, 23: 459-493.

YILMAZ 0. 2001. Seismic Data Analysis: Processing, Inversion, and Interpretation of Seismic Data - 2nd ed., Soc. Expl. Geophysicists, Volumes I-II, 2027 pp.

ZOEPPRITZ K. 1919. Erdbebenwellen VII b. Über Reflexion und Durchgang seismischer Wellen durch Unstetigkeitsflächen. Nachrichten von der Königlichen Gesellschaft der Wissenschaften zu Göttingen, Mathematisch-physikalische Klasse, 66-84. (In German).

Recebido em 24 março, 2015 / Aceito em 13 novembro, 2015

Received on March 24, 2015 / Accepted on November 13, 2015

\section{NOTES ABOUT THE AUTHORS}

José Sampaio de Oliveira holds a B.S. degree (2007) in Physics from Universidade Federal Fluminense, Brazil. Earned master dissertation (May 17th, 2010) and thesis (May 22th, 2015) to obtain the degree of Doctor in Geophysics in the Post-graduation Course in Geophysics at Observatório Nacional, Ministry of Science, Technology and Innovation, Brazil. Currently, works at Instituto Federal do Rio de Janeiro giving classes to graduation courses and developing researches on petrophysics. Member of SBGf.

Jorge Leonardo Martins holds a B.S. degree (1986) in Civil Engineering from Universidade Veiga de Almeida and a PhD (1992) in Applied Geophysics from Universidade Federal da Bahia, Brazil. Was an associate researcher at Universidade Estadual do Norte Fluminense (1993-1998), visiting researcher at the Geophysical Institute of the Czech Academy of Science (April-June/1998), post-doctoral fellow at the SW3D Consortium Project (August/1998-January/2000), visiting researcher at Universidade Estadual de Campinas (2000), associate researcher at the Pontifícia Universidade Católica do Rio de Janeiro (2001), and visiting professor at Universidade do Estado do Rio de Janeiro (2002). Currently, holds an associate researcher position at Observatório Nacional, Ministry of Science, Technology and Innovation, Brazil. Professional interests include theory and practice of seismic anisotropy, azimuthal AVO analysis, integration of seismics with well log petrophysics, multicomponent seismics, seismic inversion and seismic data processing. Member of SEG, SBGf and SPWLA Brazil Chapter. 